\title{
PENICILLIUM MARNEFFEI OSTEOMYELITIS
}

\author{
YUEN-FU CHAN, K. C. WOO
}

From Princess Margaret Hospital, Hong Kong

\begin{abstract}
We report a case of systemic Penicillium marneffei infection in a Chinese woman who presented with multiple osteolytic lesions and radiographic changes similar to those seen in other fungal osteomyelitides. This infection is often misdiagnosed as tuberculosis and has a high mortality rate. The correct diagnosis is important since early antifungal therapy is life-saving.
\end{abstract}

Penicillium marneffei is a dimorphic fungus, and a rare pathogen in man. The first description of human infection was in 1973 (DiSalvo, Fickling and Ajello 1973), and 17 other cases have been reported, all except one from South-East Asia (Jayanetra et al 1984; Pautler, Padhye and Ajello 1984; Deng and Connor 1985; So et al 1985; Yuen et al 1986; Tsang et al 1988). We describe a case of systemic $P$. marneffei infection in a woman who presented with a chronic facial ulcer and multiple osteolytic lesions. We highlight the importance of recognising $P$. marneffei as a rare cause of fungal osteomyelitis.

\section{CASE REPORT}

A 51-year-old Chinese female factory worker was admitted in August 1988 for investigation of a chronic facial ulcer and multiple painful swollen joints. She had complained of multiple itchy erythematous skin lesions since 1986. In July 1987 she had a left upper lobectomy for pulmonary tuberculosis, followed by one year of antituberculous treatment. The ulcer on her right temple had been present for one year, had slowly enlarged, and failed to respond to local treatment. Six months before admission she began to have pain and swelling of the knee, ankle, elbow, wrist and finger joints.

On admission she was pale but afebrile, with a $7 \times$ $2 \mathrm{~cm}$ ulcer on her right temple and pre-auricular region. Both hands showed ulnar deviation, with swelling and

Y. F. Chan, MRCPath, FRCPA, Consultant Pathologist K. C. Woo, FRCS Ed, FRACS, Senior Medical Officer, Orthopaedics and Traumatology

Clinical Pathology Unit and B Unit, Princess Margaret Hospital, Lai Chi Kok, Kowloon, Hong Kong.

Correspondence should be sent to Dr Y. F. Chan.

C 1990 British Editorial Society of Bone and Joint Surgery $0301-620 X / 90 / 3101 \$ 2.00$

J Bone Joint Surg [Br] 1990; 72-B : 500-3 impaired movement of wrists, finger joints and ankles. Cystic swellings were palpable over both elbows. Multiple maculopapular skin lesions 1 to $3 \mathrm{~cm}$ in diameter were most numerous on her lower limbs. She had no lymphadenopathy but her liver was palpable $5 \mathrm{~cm}$ below the rib margin. Radiographs showed multiple lucent defects in the metacarpals and phalanges of both hands, the distal ends of the forearm bones, both humeral epicondyles, the shaft of the right ulna, the right lower femur, both upper tibiae and patellae and the right lower tibia (Fig. 1). The skull and the axial skeleton were not involved.

On admission her haemoglobin was $9 \mathrm{~g} / \mathrm{dl}$ with a white cell count of $6.58 \times 10^{\%} / 1$ with $80 \%$ polymorphs, a platelet count of $244 \times 10^{9} / 1$, and an ESR of $134 \mathrm{~mm} / \mathrm{hr}$. Tests for rheumatoid factor, antibodies to doublestranded DNA and urinary Bence-Jones protein were all negative. Protein immuno-electrophoresis showed a slightly raised $\operatorname{IgG}$ level. The anti-nuclear factor was weakly positive at a titre of $1: 40$.

Biopsies were taken from the synovium of the right wrist and the facial ulcer and, after confirmation of systemic $P$. marneffei infection, courses of intravenous amphotericin B and oral 5-fluorocystosine were started. The facial ulcer healed in three weeks and the wrist and finger joints regained full movement. After two months treatment she developed a hypersensitivity reaction to amphotericin B, which was discontinued. Radiographs after seven months treatment showed that most of the osteolytic lesions had either disappeared or become much smaller.

The patient denied having travelled in South-East Asia or mainland China, but reported that some of her workmates were Vietnamese refugees. Immunological tests showed a normal number of $\mathrm{T}$-lymphocytes and a normal helper/suppressor T-cell ratio. Antibody tests for human immunodeficiency virus were negative.

Pathological findings. Histological examination of the 

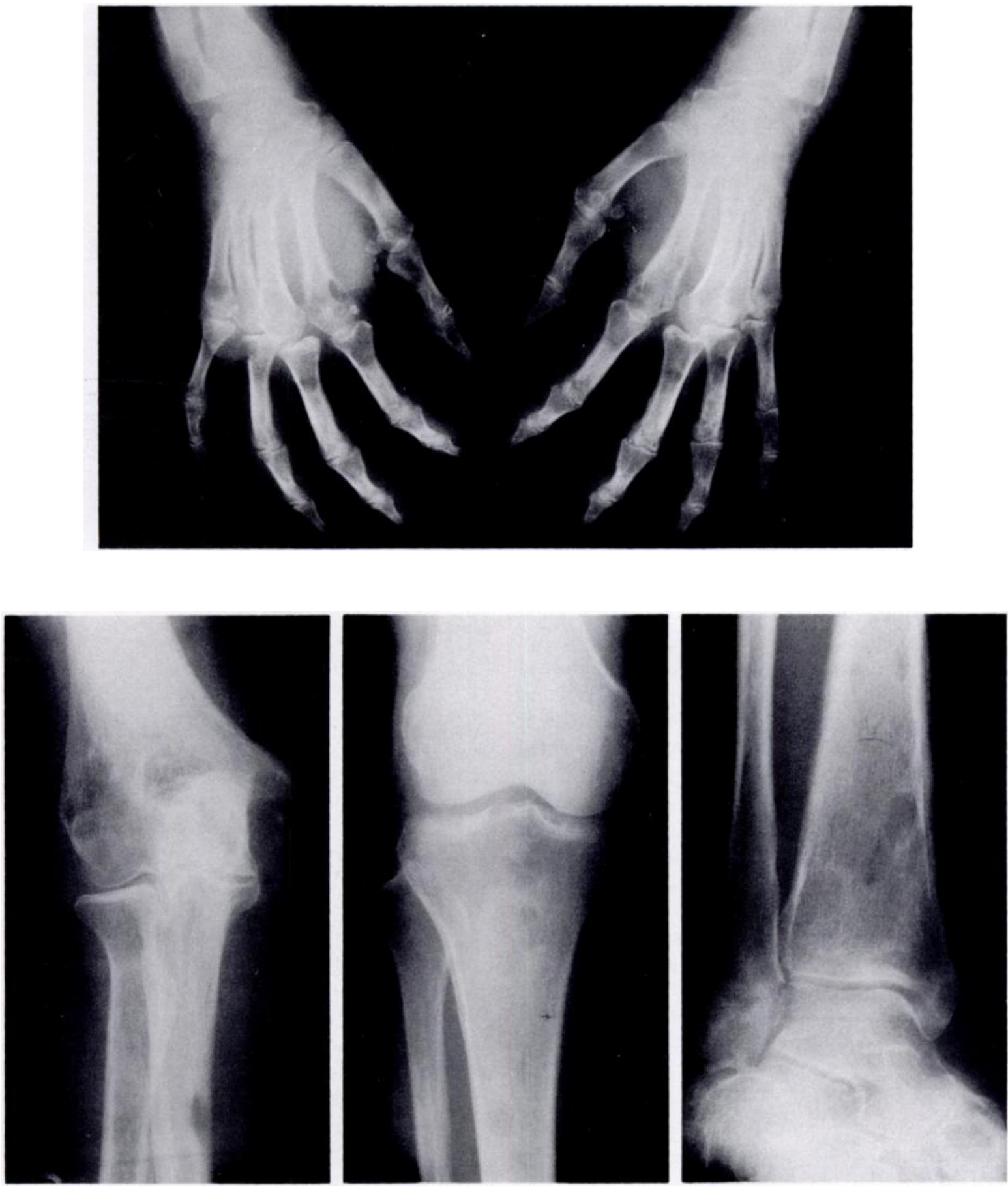

Fig. 1

Radiographs showing the radiolucent defects in a) the hands and wrists; b) the epicondyles of the right humerus and the shaft of the ulna : c) the right upper tibia ; and d) the right lower tibia and medial malleolus. 
synovial specimen showed dense fibroblastic proliferation within which there were large granulomatous foci of epithelioid cells, with a few scattered Langhans' giant cells and areas of caseous necrosis. Around one necrotic area, the epithelioid cells had developed large phagosomes, each of them engulfing up to 30 tiny yeast-like fungi (Fig. 2). Each yeast-like cell was 2 to $3 \mu \mathrm{m}$ in diameter and had a central vacuole surrounded by an eccentric rim of cytoplasm resembling a signet ring. When cultured in Sabouraud's dextrose agar at $25^{\circ} \mathrm{C}$ there was rapid growth of yellow-green fungal colonies surrounded by a red soluble pigment. A wet smear stained with lactophenol cotton blue revealed typical pencillium sterigmata.

The facial ulcer biopsy also showed numerous yeastform fungi both within and outside macrophages. Though septate forms were not well shown by methenamine silver staining, electron microscopy clearly demonstrated the presence of cross-walls in a number of the yeast cells.

\section{DISCUSSION}

$P$. marneffei is the only known dimorphic fungus among the 300 or more species of the penicillium genus. The first reported case of natural human infection involved the spleen of an American missionary with Hodgkin's disease who had travelled in South-East Asia (DiSalvo et al 1973). Since then nearly all the cases have been from South-East Asia, including five from Thailand (Jayanetra et al 1984), eight from the Guangxi Province of mainland China bordering Vietnam (Deng and Connor 1985) and three from Hong Kong (So et al 1985; Yuen et al 1986; Tsang et al 1988). P. marneffei is thus well-documented as the most likely of the penicillium species to cause severe systemic human infection. By contrast, other penicillium species usually cause superficial or single organ infection, most commonly in the form of bronchopulmonary penicilliosis (Rippon 1974).

The clinicopathological features of systemic $P$. marneffei infection have been well described. The age of patients has ranged from six to 61 years, with a male to female ratio of about 2 to 1 . Most patients were apparently healthy before the infection, but $25 \%$ of them had underlying immune disorders such as Hodgkin's disease and systemic lupus erythematosis. Clinical manifestations were protean but most patients developed fever, hepatomegaly, lymphadenopathy, soft-tissue abscesses and chronic skin ulcers.

The presentation of our patient was unusual in that she was afebrile and had no lymphadenopathy. The most striking findings were the multiple well-delineated lytic bone lesions. These showed minimal reactive sclerosis and had a predilection for small bones and bony prominences. Radiologically, the appearance and distribution of these lesions were remarkably similar to those seen in osteomyelitis caused by the more common fungal organisms such as African histoplasmosis, blastomycosis and coccidioidomycosis (Pritchard 1975; Putschar 1976; Schwarz 1984). The involvement of bony prominences in systemic $P$. marneffei infection has been reported previously in two patients, in whom the clavicle, scapula, sternum, ribs and skull were also involved (Jayanetra et al 1984). $P$. marneffei, though rare, should therefore be considered as one of the possible causative organisms in the differential diagnoses of fungal osteomyelitis.

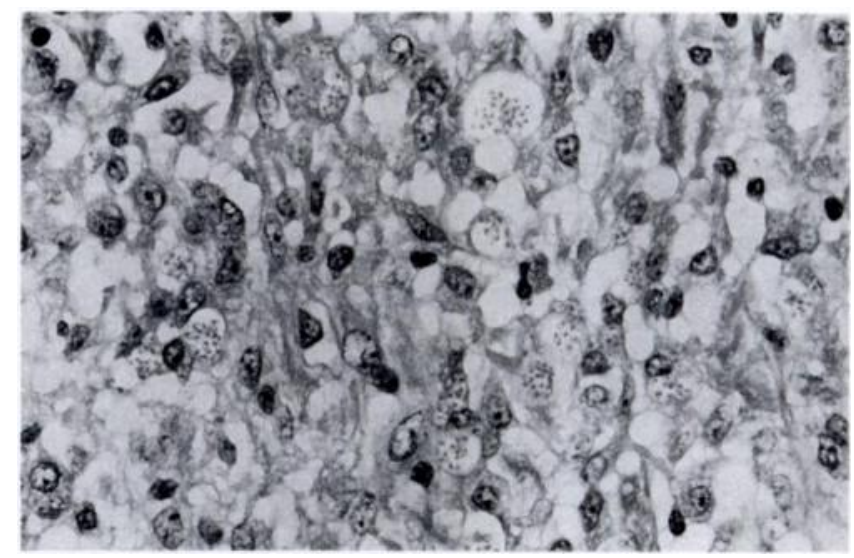

Fig. 2

Photomicrograph of the synovium showing epithelioid cells with large phagosomes engulfing numerous yeast-like fungi (Haematoxylin and $\operatorname{cosin} \times 250)$

Systemic $P$. marneffei infection has a high mortality rate and even with antifungal treatment the reported survival rate has been only about $20 \%$. In a number of cases the diagnosis has been confirmed only at autopsy. The initial diagnosis was tuberculosis in seven patients (Jayanetra et al 1984; So et al 1985; Yuen et al 1986; Tsang et al 1988); all of them were given antituberculous drugs for periods of one month to one year. This is hardly surprising, since tuberculosis is still very common in South-East Asia and the clinicopathological signs of both diseases are very similar. This point is well illustrated by our patient, who had proven history of pulmonary tuberculosis; the osteolytic lesions could easily have been misdiagnosed as tuberculous osteomyelitis.

Delay in diagnosis and treatment undoubtedly contributes substantially to the high mortality rate. It must be emphasised that pathologists should exercise extreme caution in examining sections from such patients; the only diagnostic feature, the tiny yeast-form fungi, can easily be missed. In this respect, methenamine silver staining can be of immense help in identifying the organisms. When it is given early enough, the response to antifungal therapy is rapid and gratifying, as evidenced in the few surviving patients.

From the histological point of view the only 
important fungus which may be confused with $P$. marneffei is histoplasmosis. In both conditions there are intracellular yeast-like fungi, which are similar in size. The main differentiating features are that, outside macrophages, $P$. marneffei show more variation in size: both elongated and septate forms are found (Deng and Connor 1985). When cultured in Sabouraud's dextrose agar at $25^{\circ} \mathrm{C}, P$. marneffei grows rapidly with production of a characteristic red, soluble pigment. Examination of a wet smear preparation will reveal the typical penicillium sterigmata.

Many routes of entry of the organisms into the host have been suggested, including direct inoculation, inhalation and ingestion. Our patient could have acquired the infection locally or through contact with Vietnamese workers. She had minimal pulmonary and gastrointestinal symptoms, so we consider it likely that the skin was the primary site of infection, with subsequent haematogenous spread to bones and joints. The reason for this predilection instead of the more usual involvement of the reticulo-endothelial system is unknown. Despite the small number of reported cases, we believe that $P$. marneffei infection is endemic in South-East Asia; many more cases may have masqueraded as tuberculosis.

We wish to thank $\mathrm{Mr} \mathrm{H}$. T. Au for his expert photographic assistance and Miss Windy Mo for typing the manuscript.

No benefits in any form have been received or will be received from a commercial party related directly or indirectly to the subject of this article.

\section{REFERENCES}

Deng Z, Connor DH. Progressive disseminated penicilliosis caused by Penicillium marneffei: report of eight cases and differentiation of the causative organism from Histoplasm capsulatum. Am J Clin Pathol 1985: 84:323-7.

DiSalvo AF, Fickling AM, Ajello L. Infection caused by Penicillium marneffei: description of first natural infection in man. Am J Clin Pathol 1973:60:259-63.

Jayanetra P, Nitiyanant $\mathbf{P}$, Ajello $\mathbf{L}$, et al. Penicillosis marneffei in Thailand: report of five human cases. Am J Trop Med Hig 1984: $33: 637-44$.

Pautler KB, Padhye AA, Ajello L. Imported penicilliosis marneffei in the United States: report of a second human infection. J Med Vel Mycol 1984: 22:433-8.

Pritchard DJ. Granulomatous infections of bones and joints. Orthop Clin North Am 1975: 6:1029-47.
Putschar WGJ. Osteomyelitis including fungal. In: Ackerman LV Spjut HJ, Abell MR, eds. Bones and joints. Baltimore: Williams and Wilkins, 1976, 39-60.

Rippon JW. Medical mycology: the pathogenic fungi and the pathogenic actinomycetes. Philadelphia. etc: WB Saunders Co, 1974.

Schwarz J. What's new in mycotic bone and joint diseases? Pathol Re's Pract 1984: 178:617-34

So SY, Chau PY, Jones BM, et al. A case of invasive penicillosis in Hong Kong with immunologic evaluation. Am Rer Respir Dis $1985: 131: 662-5$.

Tsang DNC, Chan JKC, Lau YT, et al. Penicillium marneffei infection: an underdiagnosed disease? Histopathology 1988:13:311-8.

Yuen WC, Chan YF, Loke SL, et al. Chronic lymphadenopathy caused by Penicillium marneffei: a condition mimicking tuberculous lymphadenopathy. BrJ Surg 1986: 73:1007-8. 